\title{
Ein Brief des Freiherrn von Stein.
}

Von

\section{Anton Becker.}

Nach der Achterklärung durch das Decret Napoleons aus Madrid vom 16. Dec. 1808, welches Freiherr von Stein, der am 24. November desselben Jahres seinen Abschied als Minister erhalten hatte, in Berlin in den ersten Tagen des Jänner 1809 erhielt 1 ), suchte er so schnell als möglich ein gesichertes Asyl zu erreichen. Er reiste am 5. Jänner von Berlin ab über Sagan, Bunzlau, Löwenberg bis Buchwald, wo er am .9. Jänner ankam und am folgenden Tage Briefe seiner Frau nebst einem Passe, den diese von dem österreichischen Gesandten, Herrn von Bombelles, erhalten hatte, empfing. Damit überschritt er die Grenze und schrieb von Trautenau an seinen Jugendfreund, den Grafen Odonell, und an den Grafen Stadion mit der Bitte, für ihn vom Kaiser in dessen Staaten ein Asyl zu erwirken 2). Dann begab er sich nach Prag; hier erhielt er zunächst eine vertrauliche Mittheilung des Grafen Odonell, dann eine amtliche Verständigung von Stadion, dass der Kaiser ihm ein Asyl gern gewähre; er ,wünsche jedoch, dass Stein die Hauptstadt von Mähren, Brünn, zum Aufeuthaltsorte wählen möge, da Prag, der Sammelplatz vieler durch das Unglück der Zeiten brotoder dienstlos gewordenen Personen und aller preussischen Civil- und Militärbeamten, grösstentheils sehr achtbarer, aber nicht selten unvorsichtiger Leute, ihm die wünschenswerthe Ruhe nicht gewähren würde " 3). Noch Ende Jänner reiste Stein nach Brünn, wo er im Kreise der Seinen bis Mitte Juli 1809 lebte.

1) Pertz, Das Leben des Freiherrn v. Stein (Berlin 1850) II, 319.

2) ibid. 324 .

o) ibid. 325 . 
Man hat geklagt, dass wir über keine Zeit ron Steins Leben so mangelhaft unterrichtet sind, wie gerade über die seines Brünner Aufenthaltes 1); man fand es bedauerlich, wenn auch nicht überraschend, dass sich aus dieser Zeit so wenig Nachrichteu von ihm finden und erklärte es damit, dass er es vermuthlich für unvorsichtig hielt Briefe zu schreiben ${ }^{2}$ ).

Mit Rücksicht darauf erscheint es mir nicht unpassend, einen Brief Steins aus dieser Zeit, der mir in Archiv des k. k. Ministeriums des Jnnern in Wien zuhanden kam, zu veröffentlichen; derselbe ist an geheimen Kriegsrath Kunth gerichtet, der nicht bloss Steins Geldangelegenheiten besorgte, sondern auch sich lebhaft bemühte, durch Vermittler auf die französischen Machthaber zu seinen Gunsten einzuwirken ${ }^{3}$ ), und lautet folgendermassen:

Brünn, am 7. Mai 1809.

Noch sind wir ruhig hier, und durch grosse Streitkräfte geschützt. - Ein grösseres Uebergewicht erhält bei mir der Gedanke, die Wanderungen zu endigen und hier alles abzuwarten, was das Schicksal ausspricht und auch $\mathrm{Ihm}$ mich zu überlassen, wenn er hier mich erreichen sollte. Denn ich glaube sein Hauptzweck war; mich zu entfernen und andere zu schrecken, da dieser erreicht ist, so hat die Sache für ihn weiter kein grosses Interesse mehr. Und wass kaun am Ende mir für grosses Unheil zugefügt werden, indem kein Grund vorhanden ist zu besonderer persönlicher Misshandlung. Sollten die grossen und edelen Zwecke, die man hier mit so ausserordentlicher Anstrengung und Aufopferung zu erringen strebt, nicht errungen werden, so gestehe ich, so bleibt nichts mehr zu erwarten übrig und mein ganzes Leben wird in einem trüben Hinbrüten unter Vergangenheit und Gegenwart und in Verrichtung der animalischen Functionen bestehen" 4 ).

Dieser Brief ist nach zwei Seiten hin von grossen Interesse; zunächst durch den grossen Optimismus, den hier Stein mit Rücksicht auf sein Verhältnis zu Napoleon zum Ausdruck gibt; dann durch die Resignation, mit der er sein Schicksal erwartet.

1) Seeley, Stein, Sein Leben und seine Zeit. Deutsch v. Emil Lehmann. (Gotha 1885) II, 309.

2) ibid. 310 .

8) Pertz II, 341.

4) Archiv d, k. k. Minist. d. Innern 1809. Nr. 28 fasc. 137. Ich ergreife an dieser Stelle die Gelegenheit, Herrn Archivdirector Dr. Thomas Fellner, sowie Herrn Dr. Rich. Schuster für die liebenswürdige Unterstützung meiner Arbeiten im obigen Archive meinen verbindlichsten Dank auszusprechen. 
Was das erstere anbelangt, so ist dieser Optimismus um so anffallender als darin noch immer jene Ansicht zur Geltung kommt, die Stein selbst ausgesprochen hat, als er unter der Einwirkung der Veröffentlichung seines aufgefangenen Briefes im Moniteur sein Entlassungsgesuch am 18. October 1808 beim Könige eingebracht hatte, nämlich dass Napoleoṇ sich beruhigen werde, wenn der König ihn seines Postens enthebe 1). Nun war aber seit dieser Zeit schon soviel gegen Stein Feindliches von Seite des französischen Kaisers geschehen, dass man annehmen könnte, Stein habe diese Ansicht fallen lassen. Zwar lässt sich das, was unmittelbar nach der Veröffentlichung jenes fatalen Briefes geschah, noch ganz als Begründung von Steins Ansicht anführen. Wenn Stein den Brief Napoleons an Soult rom 10. September $1808^{2}$ ) gekannt hätte, würde er ihn nur in seiner Meinung bestärkt haben. Selbst die Drohangen der Franzosen und der französisch Gesinnten in Berlin, dem Grafen Glotz gegenüber, bei dessen Durchreise nach Erfurt, sowie deren Gewaltmassregeln gegen die Freunde Steins wie Herrn v. Troschke, die Erklärung Champagnys in Erfurt, dass Stein unmöglich im Amte bleiben könne, der heftige Ausfall Napoleons, wie es Stein ungestraft wagen könne, solche Gesinnungen zu äussern ${ }^{3}$, liessen sich in diesem Sinne deuten. Allein soviel hatte nun Stein selbst gesehen, dass das, was er in seinem Entlassungsgesuch für "wahrscheinlich" hielt, sich nicht bewahrheitete; denn Napoleon beschäftigte sich trotz des spanischen Krieges lebhaft mit Stein; ich bin sogar der Ansicht, dass der Kaiser gerade in Spanien den Plan gefasst hat, Stein zu vernichten ${ }^{4}$ ), nicht so sehr um ein Beispiel zu statuieren, sondern weil er - man muss sagen instinctmässig - die Gefährlichkeit Steins fühlte. Hatte er auch durch sein Feldherrntalent die spanischen Scharen geschlagen und zurückgeworfen, erschien auch für den Moment der spanische Krieg beendet, so hatte doch Napoleon mit seinem scharfen Blick die Gefährlichkeit dieses Volkswiderstandes erkannt, wenn er auch in seinem Bulletin vom 13. November $1808^{5}$ ) aus Burgos sagt, das diese erregten Volksmassen

1) Pertz II, 260.

2) Correspondence XVI, 503. ,J' ai demandé, qu' il (Stein) fût chassé du Ministère, sans quoi le roi de Prusse ne rentrera par chez lui. De plus j' ai fait mettre le sequestre sur les biens en Westphaliec.

8) Pertz II, 258.

4) Gegenuber Seeley, der der Ansicht ist, dass Napoleon von Anfang an darauf dachte.

5) ,Il faudrait, que les hommes comme M. de Stein, qui a dèfaut des troupes de ligue, qui n' ont per resister à nos aigles, méditent le sublime projet de lever 
regulären Truppen wenig Widerstand leisten könnten. Aus diesem Bulletin liest man förmlich den Gedankengang, der Napoleon auf Stein brachte. Als Minister, der sich einem eventuellen Anschlusse an Oesterreich mehr als günstig zeigte und der auf den König Einfluss genug besass, um ihn vielleicht zu einer Theilnahme an dem bevorstehenden Kriege zu veranlassen, war Stein von Napoleon am selben Tage ${ }^{1}$ ) unmöglich gemacht worden, an welchem Prinz Wilhelm in Paris die Convention unterzeichnete, durch welche, wie der Moniteur bemerkte, „alle zwischen Frankreich und Preussen noch bestandenen Misshelligkeiten beigelegt worden waren" 2). Des Staates Preussen war man also ziemlich sicher. Wie gefährlich konnte aber eine „Erhebung der Massen" in Norddeutschland, wo es ja allerorts gährte, an geheimen Gesellschaften nach Versicherung französischer Spione kein Mangel war, gerade in dem Augenblicke werden, wo der Krieg nit Oesterreich vor der Thüre stand, besonders dann, wenn man in diesen Volksaufstand unter guter Leitung ein gewisses System brachte. Und wer war hiezu geeigneter als Stein, der in Westphalen seine Güter besass, der überall unter dem Landadel, wie an den Höfen Beziehungen hatte und der in dem aufgefangenen Briefe meint, es sei rathsam die täglich zunehmende Erbitterung in Deutschland zu nähren. Stein musste also ganz unschädlich gemacht werden. So kam die bekannte Achtserklärung; wenn Napoleon jemanden zum Feinde Frankreichs und des Rheinbundes erklärte, so war dies gewiss weder für den Betreffenden eine blosse Drohung, noch für andere nur ein Schreckmittel; besonders hätte die Stelle: „Der besagte Stein wird überall, wo er durch unsere oder unserer Verbündeten Truppen erreicht werden kann, persönlich in Haft genommen" 3 ), Stein überzeugen können, dass es Napoleon nicht bloss darum zu thun war, ihn ,zu entfernen und andere zu schrecken". Doch scheinen gerade die Umstände, unter welchen Stein von dem Decrete in Kenntnis gesetzt wurde, mildernd auf dessen Auffassung eingewirkt zu haben. Der neue französische Gesandte, Herr v. St. Marsan, sandte nämlich bei seiner Ankunft in Berlin durch einen guten Bekannten Steins, den holländischen Gesandten v. Goldberg, das Decret demselben zu, mit dem Bedeuten, dass er alle Beziehungen zu Preussen abbrechen müsse, wenn er Stein noch in Berlin treffe;

les masses, fussent tèmoins des malheurs qu' elles entrainet et du peu d' obstacles que cette resource peut offrir à des troupes règlèes. Seeley II, 240.

1) 8. September 1808.

2) Moniteur vom 10. September 1808.

8) Pertz II, 319. ,Le dit Stein sera saisi de sa personne partout où il pourra être atteint par nos troupes ou celles de nos alliés ‘ 
fills er gleich abreise, nehme er an, er sei bereits abwesend 1). Stein hat vermuthlich aus dieser privaten Rücksicht St. Marsans den Schluss gezogen, dem er in seinem Brief Ausdruck gibt. Dazu kam noch ein Brief des Fürsten Wittgenstein, den Stein in Prag am 20. März 1809 erhielt. Es war die Antwort anf die Aufforderung Steins, sich über zwei Briefe zu erklären, die Wittgenstein an ihn und Goltz geschrieben und worin er sich die Fortsetzung eines chiffrierten Briefwechsels verbeten hatte; diese Briefe waren im Moniteur gleichzeitig mit der Achtserklärung abgedruckt worden. Wittgenstein meint in diesem Briefe, er vermuthe, dass die Beschlagnahme der Güter nicht lange dauern würde, Stein hätte nicht Berlin verlassen, sondern ruhig dort bleiben sollen, da man seine Auslieferung nicht gefordert haben würde; Napoleon hätte durch die Massregel gegen ihn eigentlich die Beförderer eines neuen österreichischen Krieges zu schrecken beabsichtigt ${ }^{2}$ ). Das war nun die Ansicht an der Stein festhielt, trotzdem er von der genauen und strengen Achtvollstreckung sowie an dem Schicksale seiner Freunde und seiner Schwester, welche nach dem misslungenen Dörnbergischen Aufstande nach Frankreich als Gefangene gehen musste, Kenntnis hatte. Andere theilten nicht diese Meinung; vor allem nicht die österreichische Regierung, welche von dieser Stimmung Steins Kenntnis hatte; man liess ihm einen ,nicht amtlichen vertraulichen Wink" geben, ,sich über das Schicksal, welches ihm bevorstehe, nicht zu täuschen, sondern, wenn die Gefahr sich nähere, ihr auszubiegen" " 3 ).

Noch auffallender als dieser Optimismus erscheint die Resignation, die gewissermassen das Leitmotiv dieses Briefes bildet, besonders wenn man bedenkt, diss eine solehe Stimmung bei Stein weder vor noch nachher nachweisbar ist. Doch ist auch sie begründet, sobald man eine Reihe von Umständen ins Auge fasst.

Stein war schon als Minister einer der eifrigsten Parteigänger des österreichischen Krieges gewesen ${ }^{4}$ ). Als er nun im Jänner 1809 in Prag weilte, erhielt er von zwei Seiten Andeutungen, welche in ihm den Glauben erweckten, dass man iu Oesterreich beabsichtige, ihm eine Rolle in dem bevorstehenden Kampfe zuzuweisen. Zuerst schrieb Gentz, der damals als Privatmann, aber inımer in der regsten Verbindung mit den einflussreichsten Personen in Prag lebte, am 23. Febr. 1809 an Stein, dass er sich freue, „dass die österreichische Regierung

1) ibid. 321.

2) ibid. 330 .

s) Archiv d. Minist. Innern. Hager an Ezh. Rainer, Ofen, den 13. Mai 1809.

4) Wertheimer, Geschichte Oesterreich und Ungarns im orsten Jahrzehnt d. 19. Jahrhunderts II, 266, Pertz II, $205 \mathrm{ff}$. 
den Auftrag an die Behörden erlassen habe, Stein mit der grössten Auszeichnung zu behandeln und fährt dann fort: „Alle die, welche noch wissen, auf welchem Wege Heil und Rettung zu finden wäre - wenn sie gleich längst daran verzweifelt, dass man es auf diesem Wege suchen werde, - verehren in Eu. Excellenz den Patriarchen, das Oberhaupt ihrer Kirche; aus diesem Standpunkte habe ich wenigstens, und haben die, welche mit mir gleich denken, Sie schon seit mehreren Jahren betrachtet; die letzten Begebenheiten haben unserem Glauben das Siegel aufgedrückt. - Und ich meines Theils, erkläre hier, dass, wenn es mir heute gelänge, Eu. Excellenz die Dictatur (im eigentlichen, altrömischen Sinne des Wortes) über alles, was zur Rettung von Deutschland unternommen werden müsste, zusprechen zu lassen, ich morgen, mit meinem Tagewerk zufrieden, über den Ausgang und über die Zukunft beruhigt, die Welt verlassen wollte" 1 ). Am 24. Februar schrieb ihm Stadion amtlich: „Dass der Kaiser sich freue, in seinen Staaten einen Minister aufzunehmen, der eben sosehr durch die seinem Könige geleisteten Dienste als durch das für ihn daraus geflossene Unglück ausgezeichnet sei; Stadion fügte hinzu, ihm persönlich sei es lieber, Stein in grösserer Nähe, nur eine kleine Tagereise von Wien, als in der Entfernung von Prag zu wissen" 2). Nichts ist daher natürlicher, als das Stein die Erwartung hegte, zu irgend einer Thätigkeit berufen zu werden. Er hatte in Brünn angelangt eine Denkschrift über die preussischen Verhältnisse geschrieben und sie Ende Februar an Stadion geschickt ${ }^{3}$ ); allein Woche auf Woche vergieng, ohne dass ihm von der österreichischen Regierung eine Aufforderung zugekommen wäre, weder die nach Wien zu kommen, noch auch die, eine besondere Mission zu übernehmen. Das war für den thatenlustigen Mann, der jetzt Musse genug hatte seine Ideen auszuarbeiten, eine harte Enttäuschung, so zur Unthätigkeit verdammt zu sein. Als der Krieg erklärt war, suchte er am 17. April um die Erlaubnis an, nach Wien kommen zu dürfen ${ }^{4}$ ). Dieser Brief kreuzte sich auf seinem Wege mit einem von Gentz gleichen Datums, welcher diese Erlaubnis enthielt und worin Gentz sein bisheriges Schweigen dadurch zu entschuldigen sucht, dass er zu erkennen gibt, er könne sich das Benehmen des Wiener Hofes nicht erklären ${ }^{5}$ ). Thatsächlich bleibt es auch für uns unerklärlich; die Verwendung von Steins Energie und

1) Pertz II, $331 \mathrm{f}$.

2) Pertz II, 325.

9) ibid. 357 .

4) ibid. 358 .

5) ibid. 359 .

Mittheilungen XVI. 
Fühigkeiten, sowie seines Einflusses in Norddeutschland wäre für den Krieg von unberechenbaren Folgen gewesen. Es ist dies um so auffallender, als die österreichische Regierung anfangs die Absicht hatte, mit der Erregung der Gemüther in Norddeutschland zu rechnen ${ }^{1}$ ). Ob Vergesslichkeit oder die Furcht, das Volk zum Selbsthandeln zu ermuthigen, wie Seeley meint ${ }^{2}$ ), diese Absicht unausgeführt liessen, ist nicht gewiss. Die Verstimmung Steins über seine gezwungene Unthätigkeit zeigt sich schon in dem Antwortschreiben an Gentz vom 20. April. Er wolle sich nicht vordrängen, man habe ja nichts für ihn thun wollen, ihm nur den "Gebrauch des Feuers und Wassers" erlaubt. Das Schweigen der österreichischen Regierung habe seine Meinung bestätigt, dass seine Lage es fordere, sich ruhig zu verhalten und nicht die zudringliche lästige und zwecklos-thätige Rolle eines nach der Wiederherstellung seines Zustandes jagenden Emigranten zu übernehmen. Er kann aber nicht umhin, am Schlusse Winke zu geben, wie es gelingen könnte, Preussen zur Theilnahme am Kriege zu bewegen ${ }^{3}$ ).

Diese Verstimmung Steins steigert sich immer mehr, wie aus dem Brief an den geh. Staatsrath von Schön vom 30. April ${ }^{4}$ ) zu ersehen ist. „Wir leben in den Zeiten der Aufopferung und des Märtyrerthums und man muss sich dieser Anforderung nicht entziehen". Aus Preussen hörte er auch wenig Errfreulıches. Freiherr von Troschke schrieb ihm aus Ostrowe in Preussisch-Schlesien am 28. April 18095): „Officiell ist es, dass nicht nur der Adel, wie das vorige mal ... (unleserlich)... sondern der General-Landessturm aufgeboten wird. Welche Summen wird dieses wieder in Berlin kosten, wenigstens werden 13 CavallerieRegimenter aufgestellt werden müssen. Der ganze Ackerbau wird liegen bleiben müssen; ebenso wahr ist es, dass sich Bürgerkriege zwischen den Deutschen und Polen entspinnen. Es ist schon zu blutigen Scenen gekommen, Bürger gegen Bürger haben gefochten, es sind Menschen geblieben. (Auf einem kleinem, von einem anderen Brief abgeschriebenen Zettel): Vor ungefähr 36 Stunden ereigneten sich Dinge und folgten Veränderungen allhier, die jederman in Staunen setzten; die Feindseligkeiten haben ihren Anfang genommen, jedoch Dato nicht in unserem Lande, sondern auf anderen Punkten, die ịch jetzt nicht zuverlässig erfahren habe. Vorgestern abends be-

1) Vortrag Stadions v. 27. September 1808 Wertheimer l. c. II, 267.

2) Seeley Il; 308.

s) Pertz II, 363-4.

4) ibid. 365 .

5) Archiv d. Minist. d. Innern. 1809 Nr. 28 fasc. 137. 
nachrichtigte der Prinz von Pontecorvo, sonst Bernadotte genannt, unseren König officiellement, dass die Russen gemeinschaftlich mit den Polen in Galizien eindringen und der Kaiser dieser Nation sich folglich zum Alliierten des französischen Kaisers declariert habe. (Im Briefe): Hier kommt alles wieder auf den alten Fleck; die Militärexcesse fangen von neuem auf dem alten Fusse an; noch ist der brave Ob. L. G . . . n noch nicht zurück und schon hat sich ein neuer Aufstand ereignet. Die Spaltung muss bleiben, so lange man die Conscription nicht einführt, weil nur solche zum Dienste kommen, welche eine Vereinigung nicht bewirken könuen. Bei den jetzigen Aushebungen sieht man nach dem Zollmass, und lässt geschickte Leute, wenn sie das Mass nicht haben, zurück. Wir leben ohne alle Kraft und Energie, jeder sucht seinen Posten zu verlieren oder schimpft, wenn es nicht nach seinem Kopfe geht und dies ist dann natürlich von der vortheilhaftesten Wirkung".

So ist es erklärlich, dass Steins Verstimmung immer sich steigerte; den Höhepunkt derselben lesen wir in dem Briefe vom 7. Mai an Kunth; sie scheint den ganzen Monat Mai angehalten zu haben; einen Nachklang finden wir in dem Briefe an denselben vom 21. Mai 1): „Da ich von allen öffentlichen Geschäften entfernt bin, so habe ich nur einen Wunsch, den der Ruhe". Damit war aber auch diese Resignation zu Ende. Mochten vielleicht der Sieg von Asparn und der Geist der Bevölkerung, dessen Zeuge Stein war und den er nicht genug zu rühmen weiss, dazu beigetragen haben. Der Misserfolg der Schlacht bei Wagram zwang ihn zwar in Troppau ein sicheres Asyl zu suchen, war abur nicht im stande, ihm diese muthige und zuversichtliche Stimmung zu benehmeu. Als gegen Ende Juli die Nachricht von der englischen Expedition kam, da arbeitete er mit einem wahren Feuereifer an dem Projecte einer Erhebung Deutschlands und setzte seine Pläne dem Prinzen von Oranien und Gentz in rasch aufeinander folgenden Briefen auseinander, bis der Wiener Friede diesen Unterhandlungen ein Ende machte.

So erscheint dieser Brief als ein Belegstück für eine Krise im Seelenleben Steins, die im starken Contrast steht zu dessen thätiger und zielbewusster Natur; Dank seiner Energie hatte er sie rasch überwunden; den Kopf hängen zu lassen, dazu war Stein nicht geeignet.

Noch einmal während seiner Verbannung kehrte Stein nach Brünn zurïck. Es war unmittelbar vor dem Frieden. Graf Laszansky meldet am 11. October 1809 Steins Ankunft an Hager und frägt an, „ob sein

1) Pertz II, 620 f. 
Briefwechsel beobachtet werden soll oder nur derjenige der bei den Spitälern zurückgebliebenen französischen Employers", welch' letztere Massregel er empfiehlt. Hager berichtet darüber am 27. November 1809 in Ofen an den Kaiser, der am 22. December 1809 das Actenstïck folgendermassen erledigte: „Solange Baron Stein sich nicht durch sein Benehmen verdächtig macht, ist kein Grund vorhanden, in seinen Briefwechsel Einsicht zu nehmen. Sie werden dagegen dem Hauser, den ich einer späteren Anzeige zufolge bereits nach Brünn beordnet habe, durch den Grafen Laszansky auftragen lassen, die Correspondenz der bei den Spitälern in Brünn oder sonst zurückgebliebenen französischen Employers und derjenigen Einwohner, auf welchen der Verdacht einer näheren Verbindung mit den Franzosen ruhte und die ihm der mährische Gouverneur zu bezeichnen hat, zu beobachten" 1). Das war nach dem Wiener Frieden!

1) Archiv d. Ministeriums d. Innern. $1809 \mathrm{Nr} .28$ fasc. 137. Hauser war ein mit der Briefmanipulation sehr vertrauter und darin oft verwendeter Mann. 